\title{
Evaluation of Electrocardiogram for Biometric Authentication
}

\author{
Yogendra Narain Singh ${ }^{1 *}$, S. K. Singh ${ }^{2}$ \\ ${ }^{1}$ Department of Computer Science \& Engineering, Institute of Engineering \& Technology, \\ Gautam Buddh Technical University, Lucknow, India \\ ${ }^{2}$ Department of Computer Engineering, Institute of Technology, Banaras Hindu University, Varanasi, India \\ Email: *singhyn@gmail.com
}

Received November 5, 2011; revised December 19, 2011; accepted December 28, 2011

\begin{abstract}
This paper presents an evaluation of a new biometric electrocardiogram (ECG) for individual authentication. We report the potential of ECG as a biometric and address the research concerns to use ECG-enabled biometric authentication system across a range of conditions. We present a method to delineate ECG waveforms and their end fiducials from each heartbeat. A new authentication strategy is proposed in this work, which uses the delineated features and taking decision for the identity of an individual with respect to the template database on the basis of match scores. Performance of the system is evaluated in a unimodal framework and in the multibiometric framework where ECG is combined with the face biometric and with the fingerprint biometric. The equal error rate (EER) result of the unimodal system is reported to $10.8 \%$, while the EER results of the multibiometric systems are reported to $3.02 \%$ and $1.52 \%$, respectively for the systems when ECG combined with the face biometric and ECG combined with the fingerprint biometric. The EER results of the combined systems prove that the ECG has an excellent source of supplementary information to a multibiometric system, despite it shows moderate performance in a unimodal framework. We critically evaluate the concerns involved to use ECG as a biometric for individual authentication such as, the lack of standardization of signal features and the presence of acquisition variations that make the data representation more difficult. In order to determine large scale performance, individuality of ECG remains to be examined.
\end{abstract}

Keywords: Electrocardiogram (ECG); Biometrics; Vitality Measures; Spoofing Attacks; Biometrics Fusion; Security

\section{Introduction}

The electrocardiogram (ECG) is a noninvasive tool used to measure irregularities present in the functioning of the heart. It is a recording of bioelectrical activity of the heart representing the cyclical contraction and relaxation of atrium and ventricle. The most important features of the ECG include the information lying in the P, Q, R, S, and $\mathrm{T}$ waves corresponding to atrial and ventricular depolarization or repolarization. Two heartbeats of a sample ECG signal and the labeled wave fiducials are shown in Figure 1. The ECG signals acquired from different individuals are heterogeneous, generally reflected in the change in morphology, amplitude and time interval of the heartbeats. The distinctiveness of ECG signals among individuals can be due to the difference in position, size and physical conditions of their hearts. The manner in which the heart's electrical phenomenon is led to individual's myocardium also plays an important role in producing unique features of heartbeat among individu-

${ }^{*}$ Corresponding author. als [1].

Different methods in support of using ECG as a candidate of biometric have been proposed in the literature [2-8]. Unlike conventional biometrics like face and fingerprint, ECG has inherent real-time vitality characteristic.

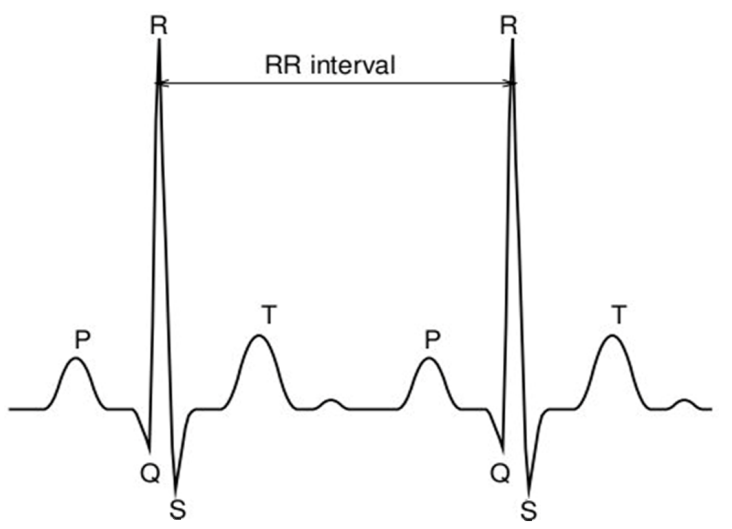

Figure 1. A sample of ECG signal that includes two heartbeats and the information lying in the $P, Q, R, S$, and $T$ waves on each heartbeat. 
The presence of vitality signs in a biometric identity ensures that it is being collected from a live and legitimate individual at the time of enrollment. Besides the vitality feature, ECG as a biometric offers other advantages to an individual authentication system. The ECG information is intrinsic to an individual so it is highly secured and confidential; it is hard to steal and impossible to mimic. The ECG is universally present among all living individuals. However, the ECG is often used by physicians to diagnose cardiac and other related ailments. Through the deployment of ECG-enabled biometric system, the identity of an individual can be verified online during ECG monitoring or offline through the medical records. This identity verification is much more useful for the protection of person identification and protection of his/her privacy about the cardiovascular condition in particular to the cardiovascular patients [9]. Although the methods of using ECG as a biometric may not offer adequate accuracy, but it has potential to supplement the information for a multibiometric system. The inclusion of ECG to a multibiometric system not only improves the system accuracy but also it improves the robustness of the system against non-live samples to be enrolled.

In this paper, we evaluate the performance of an ECGenabled biometric authentication system, both in a unimodal framework, and in a multibiometric framework where ECG is combined with the commonly used face biometric and the fingerprint biometric. Signal processing methods are used to delineate the ECG features and determine the dominant fiducials from each heartbeat. Performance of the unimodal system is evaluated on the proposed authentication strategy which uses the delineated features and taking decision about the identity of an individual with respect to the template database on the basis of match scores. We report that the system achieves the moderate performance in a unimodal framework. Further, we tested the feasibility of ECG in a multibiometric framework where it combines with the face biometric and with the fingerprint biometric as a supplement of information especially for assuring vitality detection from biometric sample. We report that the ECG can be effectively combined with the face biometric and with the fingerprint biometric for individual authentication. Transformation based score fusion technique is used to obtain the multibiometric systems and evaluated their performance using equal error rate (EER) and Receiver Operating Characteristic (ROC) curve. In order to test the operational viability of ECG-enabled biometric authentication system across a range of conditions, several concerns remain to be examined. We critically examine each of the concerns involved to use ECG as a biometric for example, lack of standardization of ECG features, variability of ECG features, individuality of ECG to a larger population and heritability of ECG features etc.
Briefly, the paper is outlined as follows. Section 2 presents a review of the existing methods that explore the feasibility of ECG as a biometric. The description of ECG-enabled biometric authentication system is presented in Section 3. The performance of the system is tested on the public database of ECG and the authentication results are given in Section 4. Section 5 describes the potential of ECG to supplement the information for a multibiometric system supported with the experimental results. The discussion on the concerns on the operational viability of ECG-enabled biometric authentication system is given in Section 6. Finally, the conclusions are noted in Section 7.

\section{Related Works}

Different studies in the recent past have shown the feasibility of ECG as a new candidate of biometric for individual authentication [2-8]. Israel et al. [2] demonstrated that ECG of an individual exhibits unique pattern. They performed ECG processing for quality check and a quantifiable metrics is proposed for classifying heartbeats among individuals. A total of 15 intrabeat features based upon cardiac physiology are extracted from each heartbeat and the classification is performed using linear discriminant analysis. The tests show that the extracted features are independent to electrode positions (e.g., around chest and neck), invariant to the individuals state of anxiety and unique to an individual.

One of the earliest studies that demonstrate the possible use of ECG for biometric application is reported by Biel et al. [4]. They conducted the biometric experiment on a group of 20 subjects where 30 features are extracted from each heartbeat. In order to reduce the amount of information the features with a relatively high correlation with other features are discarded and finally, 12 features are selected for classification. A multivariate analysisbased method is used for classification; however princeple component analysis (PCA) score plot is utilized to interpret the similarities and differences of heartbeats among individuals. Shen et al. [5] conducted the biometric experiment for identity verification using appearance and time domain features of the heartbeat. However, most of the features are extracted from QRS complex that are stable with change in the heat rates. Template matching and decision-based neural network approaches are used to quantify the identity verification rates that are reported to $95 \%$ and $80 \%$, respectively. After combining the classification approaches the result of identity verifycation is found to $100 \%$ for a group of 20 individuals.

Wang et al. [6] introduced two step fiducial detection framework that incorporates analytic and appearance based features from the heartbeat. The analytic features capture local information in a heartbeat which combines temporal and amplitude features while the appearance 
based features capture the holistic patterns in a heartbeat. To better utilize the complementary characteristics of analytic and appearance features, a hierarchical data integration scheme is presented. The method used for feature extraction is based on a combination of autocorrelation (AC) and discrete cosine transform (DCT) which is free from fiducial detection. The recognition performance of AC/DCT method is found between $94.47 \%$ and $97.8 \%$.

Recently, Singh and Gupta [7,8] explored the feasibility of ECG to aid in human identification. Signal processing methods are used to delineate ECG wave fiducials from each heartbeat. The delineation results are found optimum and stable in comparison to other published methods. The proposed $P$ and $T$ wave delineators are used along with QRS complex to extract different features from dominant fiducials of the electrocardiogram on each heartbeat. The proposed system is tested on 50 subjects ECG and the matching decisions are taken on the basis of correlation between the stored credential and the test ECG signal. The system is achieved the classifycation accuracy $\sim 98 \%$.

\section{ECG-Enabled Biometric Authentication System}

The schematic description of ECG-enabled biometric authentication system is shown in Figure 2. ECG signal which is acquired from the individuals is preprocessed for quality check. It makes the necessary correction of the signal from noise and artifact. ECG delineation in- cludes detection of $P, Q, R, S$, and $T$ waves with their dominant fiducials from each heartbeat. Feature extraction includes determination of time intervals, amplitudes and angle features from dominant fiducials of its waveforms. Finally, authentication and decision are taken by comparing the stored template and the query sample.

The heartbeats from the ECG trace are detected using QRS complex delineator which is implemented using the technique proposed in [10] with some improvements. It employs digital analysis of slope, amplitude and width information of ECG waveforms. Once the heartbeat is detected, temporal time windows are defined before and after QRS complex fiducials to seek for $P$ and $T$ wave delineations in each beat of the ECG.

\section{1. $P$ Wave Delineator}

$P$ wave represents the atrium function of the heart. The time derivative-based delineation technique is used to delineate the $P$ wave fiducials from the heartbeats [11]. The time derivative of the ECG, $y_{n T}$ at time instant $T$ is calculated using the following time difference equation,

$$
y_{n T}=-2 x_{(n-2) T}-x_{(n-1) T}+x_{(n+1) T}+2 x_{(n+2) T}
$$

where $x_{n T}$ represent the data sample of size $n$ at discrete instance of time $T$. In order to determine $P$ wave and its end fiducials, a time window is set heuristically which is extended from the beginning of QRS complex, $Q R S_{\text {onset }}$ to the beginning of heartbeat, Beat ${ }_{\text {begin }}$ as shown in Figure 3(a). The Beat $_{\text {begin }}$ fiducial can be determined by searching of first isoelectric sample prior to the start

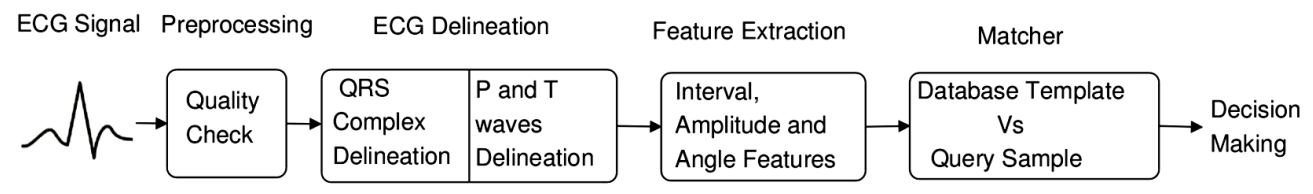

Figure 2. Schematic of ECG-enabled biometric authentication system.

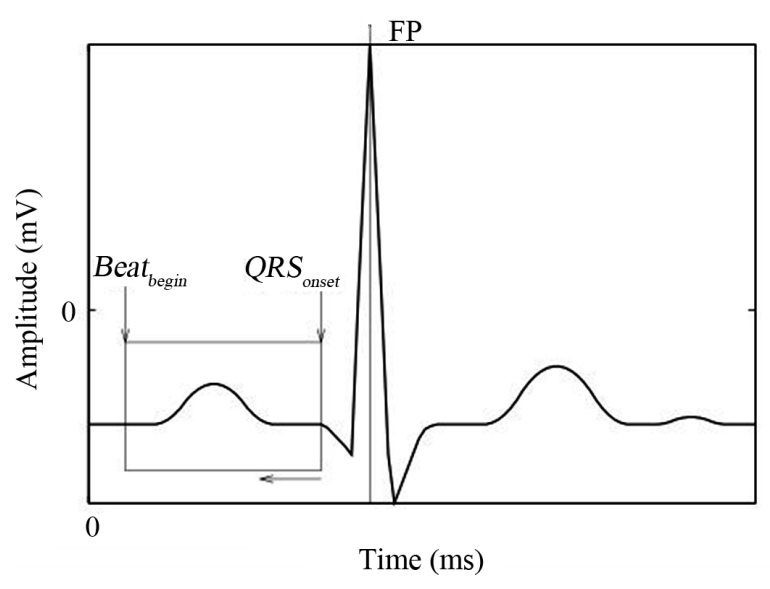

(a)

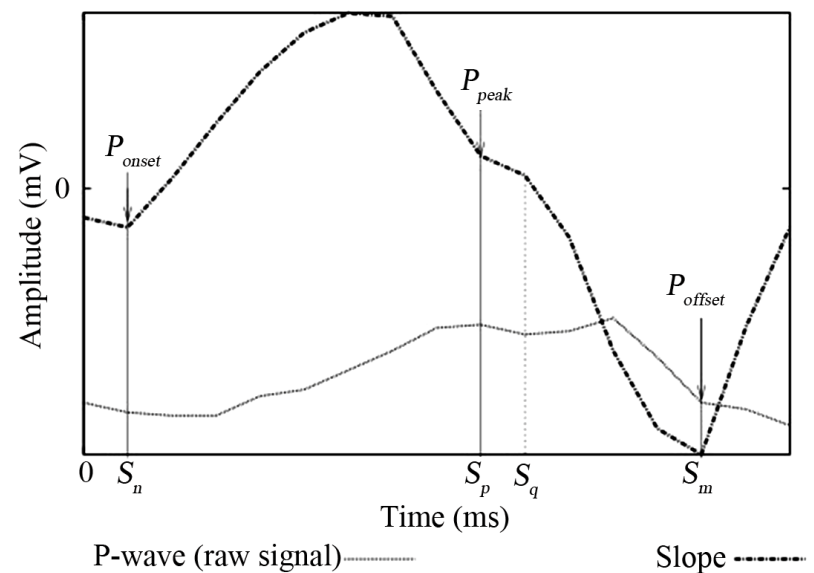

(b)

Figure 3. (a) Setting of a time window for $P$ wave delineation and (b) detection of $P$ wave fiducials in the time window. 
of atrium deflection. For the detection of $P$ waves, delineator computes the slope threshold, $\theta$. The slope threshold continuously adapts and set between the mean of most recently detected significant slopes, $\mu_{M S}$ and the mean of high frequency noise, $\mu_{H F \text { noise }}$ present in the detected beats.

$$
\theta=0.30\left(\mu_{M S}-\mu_{H F_{\text {noise }}}\right)
$$

The level of high frequency noise present in the beat can be estimated, firstly, by passing the beat to highpass filter,

$$
y_{n T}^{\prime}=x_{n T}-2 x_{(n-1) T}+x_{(n-2) T}
$$

which determines the presence of artifacts in the beat. The mean of filtered signal over a stream of samples is computed, next. Then noise metric, $H F_{\text {noise }}$ is estimated as the ratio to the maximum of averaged signal, $H F_{\text {noise }}^{M A}$ and the QRS amplitude, $h_{\mathrm{QRS}}$ using the formula,

$$
H F_{\text {noise }}=K \cdot \frac{H F_{\text {noise }}^{M A}}{h_{Q R S}}
$$

where $K$ is a constant which is set through the experiment.

The begin and the end fiducials of $P$ wave, $P_{\text {offset }}$ and $P_{\text {onset }}$, respectively are determined by tracing the ECG signal in time-reverse order within the time window. The location of $P_{\text {offset }}$ is found at sample where the slope is most negative with some adjustment. The peak of $P$ wave, $P_{\text {peak }}$ is found at the sample where the sign of slope is changed (zero crossing). After localization of peak, remaining signal is traced posteriorly for the detection of $P_{\text {onset }}$ fiducial. It is found at the sample where estimated value of $H F_{\text {noise }}$ exceeded to the slope. Found location $S_{n}$ shown in Figure 3(b) is the $P_{\text {onset }}$ fiducial.

\section{2. $T$ Wave Delineator}

$T$ wave represents ventricles repolarization. The problem with $T$ wave delineation is its repolarization cycle which terminates faster while it has lower stimulation in comparison to the noise artifact present in the beat. This makes detection of $T$ wave end fiducial, $T_{\text {offset }}$ more cumbersome. For the efficient detection of $\mathrm{T}$ wave end fiducials, the technique based on the analysis of waveform curvature is used [12]. It has corrected the signal from oscillatory patterns of reference potential using a recursive lowpass filter of following difference equation,

$$
y_{n T}=2 y_{(n-1) T}-y_{(n-2) T}+x_{n}-2 x_{(n-4) T}+x_{(n-8) T}
$$

where $x_{n}$ represents the data sample of size $n$ at discrete instant of time $T$. Prior to start of delineation process, a search window is set heuristically with respect to end position of QRS complex which is extended from
$Q R S_{\text {offest }+80 m s}$ to 1 as shown in Figure 4. The peak fiducial of $T$ wave is determined using the technique of time derivative and adaptive threshold criterion, as similar to $\mathrm{P}$ wave with some adjustments.

The end fiducials of $T$ wave are determined using the analysis of its waveform curvature that assumes the part of curvature near to its ends is convex. The signal is tracked downhill and finds the location of minimum radius of curvature as shown in Figure 5. After fixing the time difference between sample points $A, B$ and $A, C$, the minimum radius of curvature is determined by maximizing $B L$ using vector cross product between two directed line segments i.e.,

$$
B L=\frac{|A C \times A B|}{|A C|}
$$

\subsection{ECG Feature Set}

In order to validate ECG as a biometric for individual authentication, a feature set is prepared from the extracted fiducials of $P, Q, R, S$, and $T$ waves from each heartbeat [8]. The feature set contains the attributes of different classes: interval features, amplitude features and angle features which are listed in Table 1. Thirteen interval features from different times instances of the dominant fiducials of $P, Q, R, S$, and $T$ waves are computed which are shown in Figure 6(a). Amplitude features are computed relative to the amplitude of $R$ peak which is found invariant to change in the heart rate. We compute four amplitude features between the difference of amplitudes from $\mathrm{P}, Q, S$, and $T$ waves to $R$ peak as

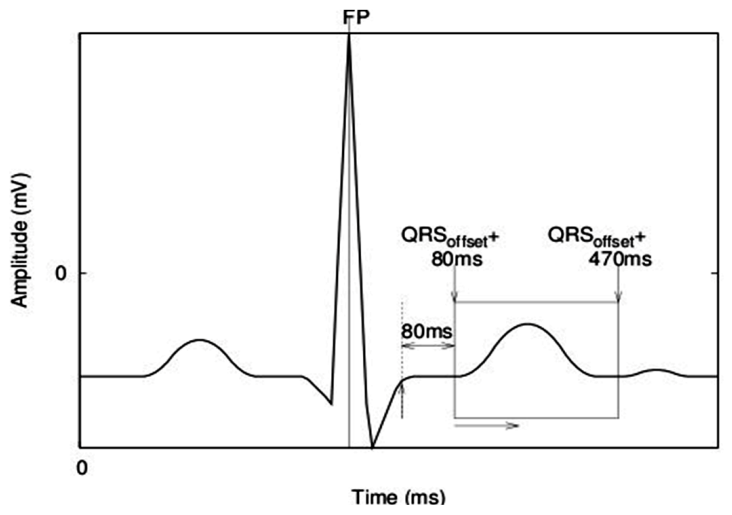

Figure 4. Setting of a time window for $T$ wave delineation.

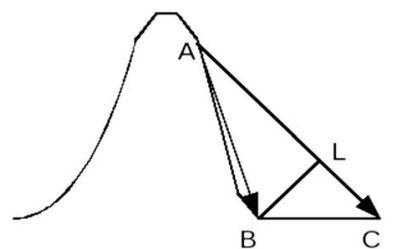

Figure 5. Detection technique used to delineate $T$ wave end fiducials. 


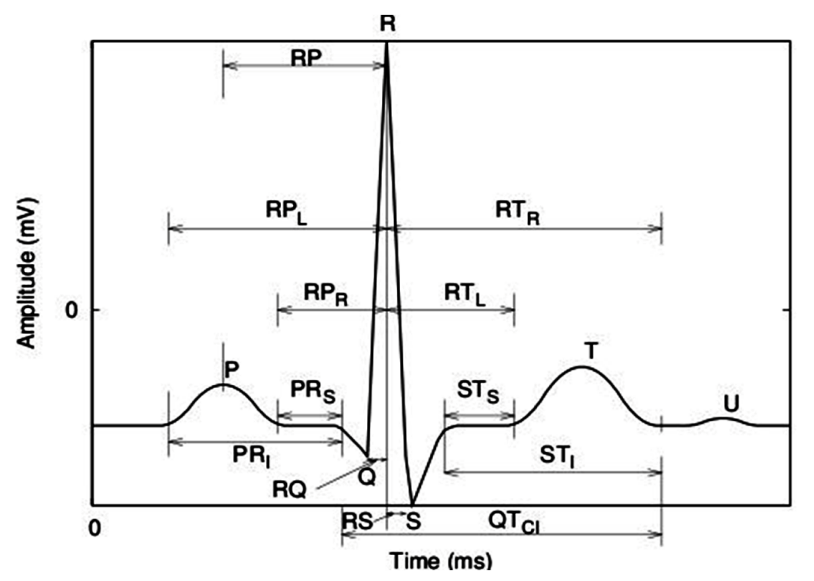

(a)

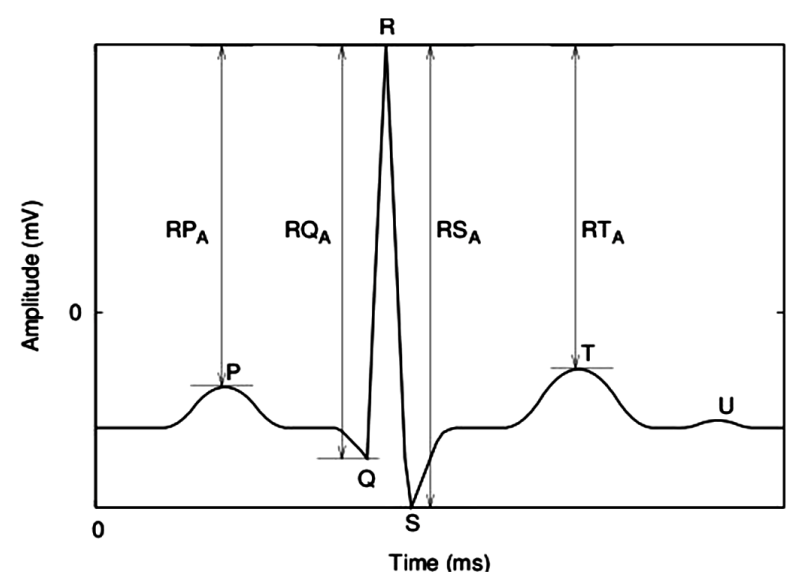

(b)

Figure 6. Considered classes of features are selected from $P, Q, R, S$ and $T$ wave fiducials of heartbeat: (a) interval features and (b) amplitude features.

Table 1. Considered classes of features are selected from ECG wave fiducials. (Here $R R$ interval is used to correct QT interval using Bazett's formula [13]).

\begin{tabular}{|c|c|c|}
\hline Classes & Features & Representations \\
\hline \multirow{13}{*}{$\begin{array}{l}\text { Interval } \\
\text { Features }\end{array}$} & $P R$ Interval & $P R_{1}$ \\
\hline & PR Segment & $P R_{2}$ \\
\hline & Corrected-QT Interval & $Q T_{C 1}$ \\
\hline & ST Segment & $S T_{S}$ \\
\hline & ST Interval & $S T_{1}$ \\
\hline & $R_{\text {peak }}$ to $P_{\text {onset }}$ Segment & $R P_{\mathrm{L}}$ \\
\hline & $R_{\text {peak }}$ to $P_{\text {peak }}$ Segment & $R P$ \\
\hline & $R_{\text {peak }} t o P_{\text {offset }}$ Segment & $R P_{R}$ \\
\hline & $R_{\text {peak }}$ to $Q_{\text {peak }}$ Segment & $R Q$ \\
\hline & $R_{\text {peak }} t o S_{\text {peak }}$ Segment & RS \\
\hline & $R_{\text {peak }}$ to $T_{\text {onset }}$ Segment & $R T_{L}$ \\
\hline & $R_{\text {peak }}$ to $T_{\text {offset }}$ Segment & $R T_{R}$ \\
\hline & RR Interval & $R R$ \\
\hline \multirow{4}{*}{$\begin{array}{l}\text { Amplitude } \\
\text { Features }\end{array}$} & RQ Amplitude & $R Q_{A}$ \\
\hline & RS Amplitude & $R S_{A}$ \\
\hline & RP Amplitude & $R P_{A}$ \\
\hline & RT Amplitude & $R T_{A}$ \\
\hline \multirow{3}{*}{$\begin{array}{l}\text { Angle } \\
\text { Features }\end{array}$} & Angle $Q$ & $\angle Q$ \\
\hline & Angle $R$ & $\angle R$ \\
\hline & Angle $S$ & $\angle S$ \\
\hline
\end{tabular}

shown in Figure 6(b). The angle class of features is related to angular displacement between different peak fiducials of $P, Q, R, S$ and $T$ waves. Here, the aim is to extract those features which are stable and consistent to the change in heart rate. The angle features are shown in Figure 7.

\subsection{Feature Normalization}

Normalization is an important issue to obtain consistent features from change in the heart rate. The heart rate varies due to change in pressure inside the heart and ventricular volume. Change in heart rate consequently changes the duration of $P$ wave, $P R$ interval and $Q T$ interval. Thus the features of $P$ and $T$ waves are normalized by dividing them to the beat length, $P R_{I}+Q T_{C I}$ while $R Q$ and $R S$ are used as raw features.

In order to investigate the effects of varying heart rate on peak fiducials of different waveforms, it is found that artial deflection do not change with the heart rate. Ventricular activation and the recovery of ventricles from stimulation are fairly invariant with change in heart rate. Therefore, raw amplitude and angle features are used in the experiment.

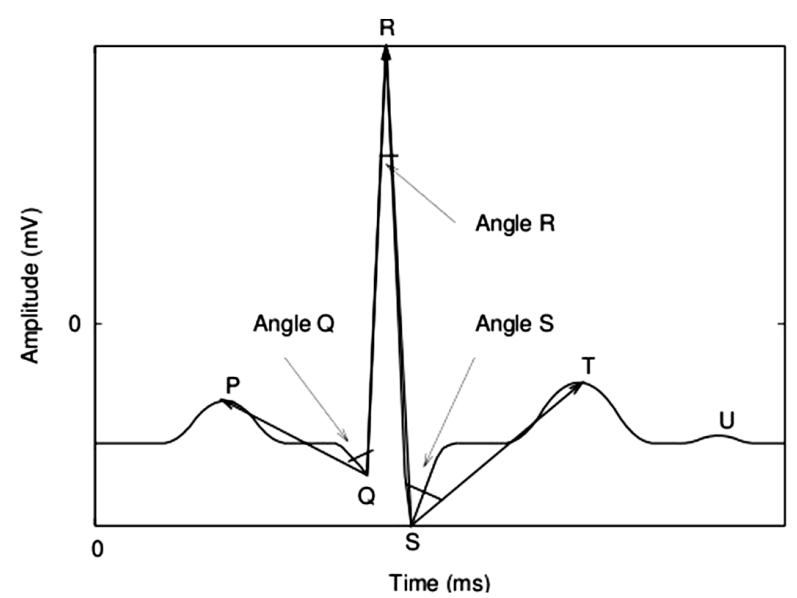

Figure 7. Angle class of features are selected from $P, Q, R, S$ and $T$ wave fiducials of heartbeat. 


\subsection{Generation of Scores from Feature Vectors}

We have adopted statistical framework approach to generate match score from the feature vectors of the template and the query samples. Consider, $G_{1}$ and $G_{2}$ are two classes correspond to genuine and impostor. Let $f$ be a feature vector denoted as $f_{1}, f_{2}, \cdots, f_{d}$ of $d$-dimension. Using Bayesian decision theory [14] the risk of misclassification such as assigning of query sample $f$ to class $G_{i}$, $i=1,2$, for which the conditional risk defined as

$$
R\left(G_{i} \mid f\right)=\sum_{j=1}^{2} L\left(G_{i}, G_{j}\right) \cdot P\left(G_{i} \mid f\right)
$$

is minimum where $L\left(G_{i}, G_{j}\right)$ is the loss incurred in deciding $G_{i}$ when the true class is $G_{j}$ and $\left(\mathrm{G}_{\mathrm{j}} \mid \mathrm{f}\right)$ is the posterior probability. In the case of $0 / 1$ loss function i.e., $L\left(G_{i}, G_{j}\right)=1$, if $i \neq j$, otherwise it is 0 , then conditional risks become the conditional probability of misclassification and the Bayesian decision rule can be simplified to maximum a posterior (MAP) rule stated as: Assign query sample $f$ to class $G_{i}$ if,

$$
P\left(G_{i} \mid f\right)>P\left(G_{j} \mid f\right) \text { for all } j \neq i
$$

Since the measures of query sample are independent conditionally to the class, therefore the required probability may be computed by combining different $P\left(G_{i} \mid f^{(k)}\right)$ for each feature vector $k$. Then the system returns a score $s^{(k)}$ where

$$
s^{(k)}=g\left(P\left(G_{i} \mid f^{(k)}\right)\right)+h\left(f^{(k)}\right)
$$

where $g(\cdot)$ is a monotonic function and $h(\cdot)$ is the error of estimation caused by the matcher [15]. If we assume that $h$ is zero then $P\left(G_{i} \mid f^{(k)}\right)$ is approximated to $P\left(G_{i} \mid s^{(k)}\right)$. Therefore, the property of match score can be defined as: If $s^{(i)}$ and $s^{(j)}$ are two scores i.e., $s^{(i)}>s^{(j)}$ then the conditional probabilities for which the query samples and the templates are from the same user is given as $P\left(G_{i} \mid s^{(i)}\right)>P\left(G_{i} \mid s^{(j)}\right)$; that means the claimed user belongs to class $G_{i}$.

\subsection{Authentication Strategy}

The computational procedure to generate the match scores from the comparison of query sample to the database template of ECG features is described as follows. Consider, an individual $i$ has an ECG data set of $t$ unit of time. The $m$ subdata sets of length $s$ unit of time $(s<t)$ are arbitrarily selected from the complete trace of an ECG. A vector of $d$-features is extracted from each subdata set. Let $P^{(i)}$ be the pattern matrix consisting of $m$ vectors of individual $i$ of size $m \times d$ which can be defined as,

$$
P^{(i)}=\left(\begin{array}{ccccc}
f_{1,1} & f_{1,2} & \cdot & \cdot & f_{1, d} \\
f_{2,1} & f_{2,2} & \cdot & \cdot & f_{2, d} \\
\cdot & \cdot & \cdot & \cdot & \cdot \\
\cdot & \cdot & \cdot & \cdot & \cdot \\
f_{m, 1} & f_{m, 2} & \cdot & \cdot & f_{m, d}
\end{array}\right)
$$

where element $f_{j, k}$ represents the $k$ th feature of $j$ th subdata set. The purpose of arbitrarily selection of subdata set is to statistically analyze the variations present in different heartbeats of an individual ECG. Consider, the population size is $n$, so there are $n$ different ECG data sets. Thus, $n$ different pattern matrices $P^{(i)}$ are generated in the database where $i=1,2, \cdots, n$.

Let an individual have a query sample $Q$ that generates the feature vector $f^{\prime}=\left\langle f_{1}^{\prime}, f_{2}^{\prime}, \cdots, f_{d}^{\prime}\right\rangle$. Statistically, the distance between the attributes of a query sample and feature vectors of a pattern matrix of an individual $i$ is computed using Euclidean distance as follows,

$$
d_{j}^{(i)}=\left(\left|f_{j, 1}-f_{1}^{\prime}\right|\left|f_{j, 2}-f_{2}^{\prime}\right| \cdots\left|f_{j, d}-f_{d}^{\prime}\right|\right)
$$

where $j=1,2, \ldots m$. The sum of Euclidean distances between attributes of feature vectors gives the distance score measure for individual $i$, as

$$
s_{j}^{(i)}=\sum_{k=1}^{d}\left|f_{j, k}-f_{k}^{\prime}\right|
$$

for all subdata sets $j=1,2, \ldots m$. In order to acknowledge the variations present in the ECG data set of an individual $i$, the mean of the distance scores, denoted as $s^{(i)}$ can be computed and determined as follows

$$
s^{(i)}=\frac{1}{m} \sum_{j=1}^{m} s_{j}^{(i)}
$$

A smaller value of distance score indicates a good match while a higher value of distance score indicates a poor match. The distance scores is then, converted to the similarity scores by subtracting each from the maximum value of the score set.

\section{Experimental Results}

The performance of the ECG-enabled biometric authentication system is evaluated on the ECG database prepared from public database of PhysioBank archives [16]. ECG recordings of 73 subjects from the class European ST-T Database, MIT-BIH Normal Sinus Rhythm Database, MIT-BIH Arrhythmia Database and QT Database are selected and the template database is prepared. Since the database only offers one ECG signal for each subject, therefore the complete record is divided in two halves such that the first half is used for preparing the template set and the latter half is used for test set.

The match scores distributions of impostor and genu- 
ine cases are shown in Figures 8(a) and (b), respectively while the equal error rate (EER) of the system is found to $10.8 \%$ as shown in Figure 8(c). The performance of ECG-enabled biometric system is shown using Receiver Operating Characteristic (ROC) curve in Figure 9. It shows that the system has genuine acceptance rate (GAR) of $59 \%$ at zero false acceptance rates (FAR). The performance of the individual system gradually increases and reached to GAR of $82 \%$ at FAR of $7 \%$. The EER result and the ROC curve show that the authentication performance of the ECG-based biometric system is low and hardly to compete the performance of the conventional biometrics (e.g., fingerprint and irises). However, ECG can be combined with other biometric and provide an excellent source of supplementary information in a multibiometric framework.

\section{Fusion of ECG with Face and Fingerprint Biometrics}

The ECG-based biometric system performs moderately in a unimodal framework. We show that ECG has the potential to supplement the information for a multibiometric system. In particular, we tested the performance of the multibiometric systems obtained from the fusion of ECG with face biometric and ECG with fingerprint biometric. The objective of the inclusion of ECG with face biometric and the ECG with fingerprint biometric is because; ECG is a physiological signal that has inherent real time vitality signs. In addition, the ECG information is intrinsic to an individual so it is hard to steal and impossible to mimic. Therefore, ECG as a biometric is robust enough against spoof attacks or falsification.

We hypothesis that ECG can be combined with face or fingerprint biometrics, effectively. Transformation based score fusion technique; in particular weighted sum rule is used to combine the match scores of different biometrics. Consider the performance of the fused biometric system is evaluated on the fusion technique that works on the assumption that weights of different identities are inversely proportional to their equal error rate (EER). Let $e_{k}$ be the EER of the biometric $k$, then weight $\left(w_{k}\right)$ assigned to biometric $k$ can be computed as

$$
w_{k}=\left(\sum_{k=1}^{t} \frac{1}{e^{k}}\right)^{-1} \cdot \frac{1}{e^{k}}
$$

where $0 \leq w_{k} \leq 1$, for all $k$ and $\sum_{k=1}^{t} w_{k}=1$. Finally, the fused score, $f_{i}$ of an individual $i$ is computed as,

$$
f_{i}=\sum_{k=1}^{t} w_{k} \cdot S_{k}^{(i)} ;(\forall i)
$$

Where $S_{k}^{(i)}$ is the similarity score of individual $i$ for biometric identity $k$ i.e., $1 \leq k \leq t$. Consequently, the total similarity measure of a multibiometric system for $n$ genuine $(G)$ scores and $n^{\prime}$ impostor $(I)$ scores is given as $\left(f_{1}, f_{2}, \cdots, f_{n}\right)_{G} \cup\left(f_{1}, f_{2}, \cdots, f_{n^{\prime}}\right)_{I}$ where

$$
\begin{aligned}
& \left(f_{1}, f_{2}, \cdots, f_{n}\right)_{G}=\sum_{k=1}^{t} w_{k} \cdot\left(G_{1}, G_{2}, \cdots, G_{n}\right)_{k} \\
& \left(f_{1}, f_{2}, \cdots, f_{n^{\prime}}\right)_{I}=\sum_{k=1}^{t} w_{k} \cdot\left(I_{1}, I_{2}, \cdots, I_{n^{\prime}}\right)_{k}
\end{aligned}
$$

and $n^{\prime}=n \times(n-1)$ for one-to-many comparison.

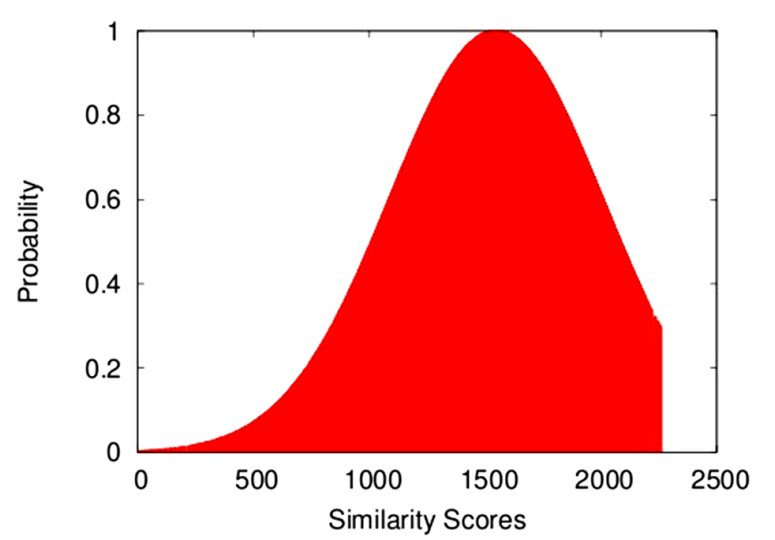

(a)

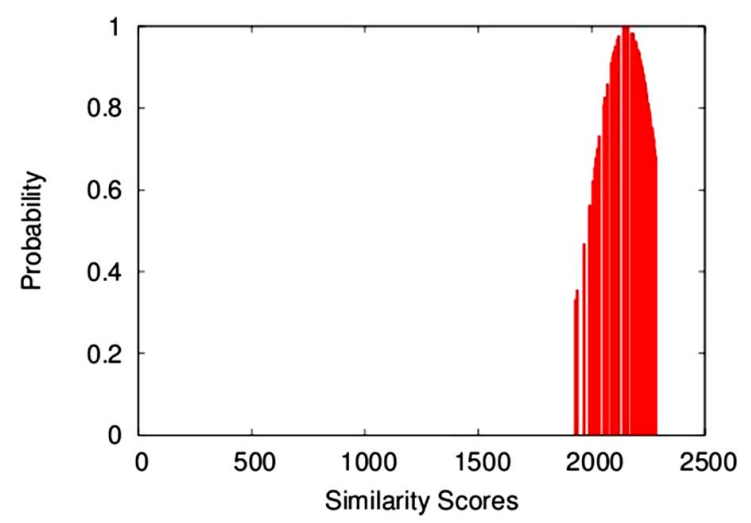

(b)

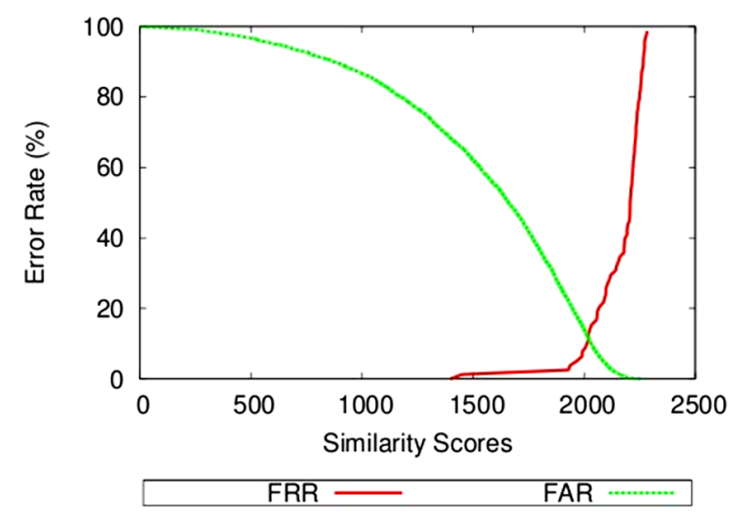

(c)

Figure 8. ECG match scores distributions (a) impostor scores and (b) genuine scores; (c) Equal error rate (EER) curve. 
We acquired the face and the fingerprint biometrics information from the public database of NIST-BSSR1 [17]. In particular scores Set 1 is used in this experiment. From this set, the fingerprint scores are generated from the comparison of two right index fingerprints and the face scores are generated from the comparison of two frontal faces by the face recognition system labeled C. Since the databases of ECG, face and fingerprint biometrics are different, therefore we can assume that an individual in ECG database has a face and fingerprint information in other database and thus creating a virtual multibiometric system. From these sets, we prepare the face and fingerprint biometrics information of 73 subjects, where the individuals are selected arbitrarily from the given list of users. The equal error rate (EER) results of the unimodal systems of face and fingerprint biometrics is found $4.52 \%$ and $2.12 \%$, respectively.

The performance of fused biometric systems of ECG with face and ECG with fingerprint are shown in Figures 9 and 10, respectively. The EER results of the aforementioned systems are reported $3.02 \%$ and $1.52 \%$, respectively for the fused system of ECG with face and ECG with fingerprint biometric. The performance of the individual systems is face and fingerprint is reported to GAR of $87 \%$ and $95 \%$, respectively for face and finger-print at zero FAR. The fused systems of ECG with face and ECG with fingerprint have achieved the performance to GAR of $94 \%$ and $96 \%$, respectively at zero FAR. The performance of the fused systems improve further and reached to GAR of $99 \%$ and $100 \%$, respectively for ECG with face and ECG with fingerprint at relatively higher values of FAR of $7 \%$. These results confirm that the ECG can be fused with face and fingerprint biometrics, effectively for individual authentication.

\section{Concerns of ECG-Enabled Biometric Authentication System}

ECG-enabled biometric methods accomplish individual authentication task through statistical analysis of the ECG signal and perform quantitative comparison of the query signal to the enrolled signals. The performance of the cited methods depends heavily on the task of data representation that means how efficiently the ECG signal is delineated. The results presented by most methods have shown the uniqueness of ECG among humans and therefore can be used for biometric applications. But most experiments are done on modest data sets acquired under controlled conditions in laboratory demonstration. To be feasible, ECG-enabled biometric system must perform the authentication task across wide range of conditions, while following issues are to be addressed.

1) Lack of standardization of ECG features.

2) Variability of ECG features.

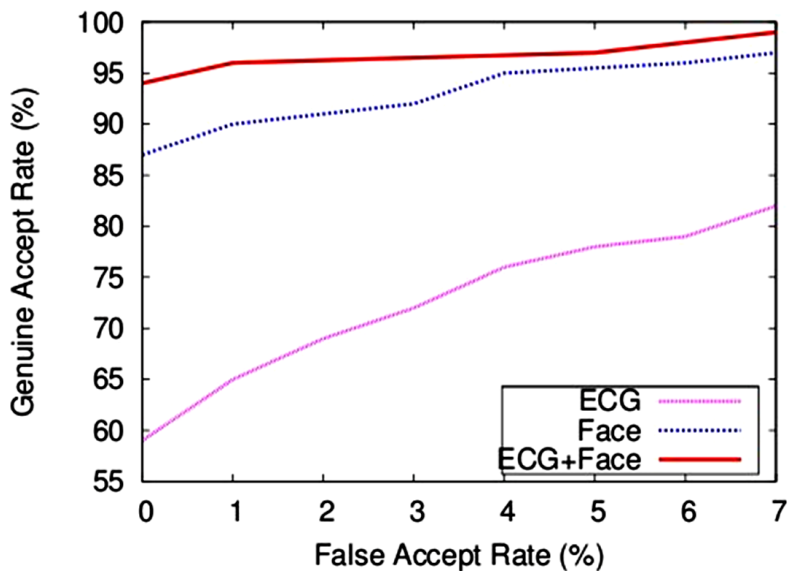

Figure 9. Performance of a multibiometric system after fusing ECG with face biometric.

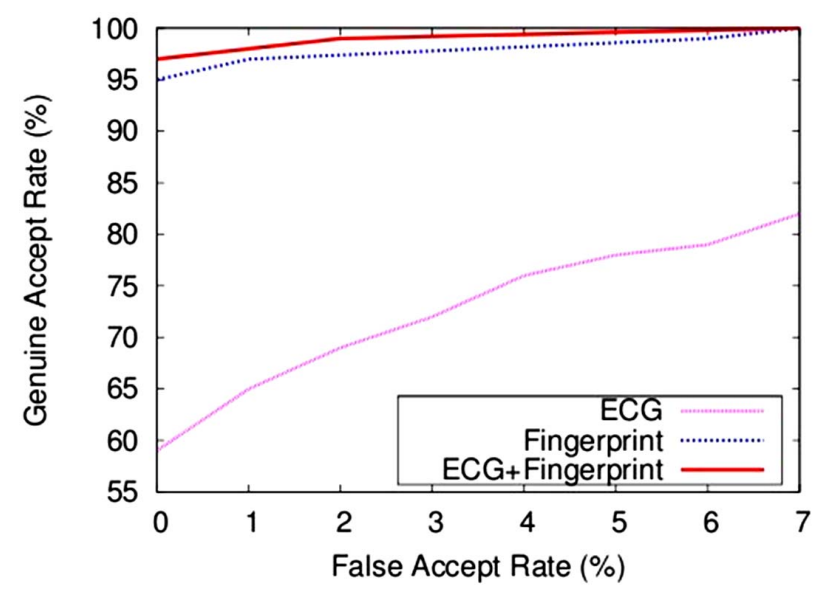

Figure 10. Performance of a multibiometric system after fusing ECG with fingerprint biometric.

3) Individuality of ECG.

4) Heritability of ECG waves.

5) Customization of ECG-enabled biometric system.

\subsection{Lack of Standardization of ECG Features}

Most methods that describe ECG as a biometric are based on the temporal or heuristic selection of wave boundaries on time and amplitude domain. Using the information of local maxima, local minima and zero crossings at different scales, the methods identify the significant fiducials of the ECG waves. The effectiveness of these methods rely on the accuracy of the detected fiducials which is a challenge due to the lack of any standardized definition of effective localization of ECG wave boundaries.

To use ECG as a biometric, the knowledge utilized by researchers is mainly based on medical findings of ECG information and they analyze the ECG on the approximate information of medical dominant fiducials. Although, the approximate localization of different waves fiducials may sufficient for clinical diagnosis but this 
information is certainly not sufficient for biometric decision making. Because the applications of using ECG as biometric expect the exact localization of ECG waves fiducials and a slight variation in localization of these fiducials may cause a misclassification i.e., false acceptance or false rejection errors over a large data sets.

\subsection{Variability of ECG Features}

Another concern that limits the use of ECG for biometric applications is the variability of cardiac rhythm within the subjects or between the subjects. Heart rate varies with individual's physiological and mental conditions. Stress, excitement, exercise and other working activities may have impact on the heart rate and it can elevate. It is to be noted that other than changes in the rhythm of heartbeat, the morphology of an ECG remains unchanged. The changes in the heart rate consequently varying different patterns of the ECG, like RR interval, PR interval, and QT interval. These features are carefully transformed and make them free from the variations in the heart rate before using for biometrics applications [8]. An interesting discussion on the link between the individual's physiology and the heart rate has been given in [8]. Aging may impact heartbeat mainly up to the age of adolescence. Some features of heartbeat may vary due to the progressive change in individual's anatomy to the adolescence ( $\sim 16$ years of age).

Other factors like presence of artifacts and emergence of irregular beats may cause the changes in the morphology of the heartbeat. The dominant frequency of powerline interference $50-60 \mathrm{~Hz}$ can distort the ECG morphology while the presence of ectopic beats or premature beats can make the individual's classification harder. Most methods have employed the techniques that have been tested on a limited class of ECG patterns. Therefore, it is needed that the method can be extended to any number of morphologically distinct ECG waveforms for the operational viability of ECG-enabled biometric authentication system.

\subsection{Individuality of ECG}

Biometric individuality is a major concern to assess the performance of a biometric system. Individuality of a biometric refers the likelihood of interclass variability and intraclass similarity of testing patterns observed in a target population. For example, individuality of ECG refers that up to what extent the ECG patterns are scalable in a target population of sufficiently larger size. Statistically, the problem of individuality of ECG is yet to be studied.

Unlike to that, the feasibility of ECG for biometric use of individual authentication explored by most methods has been tested on a limited data size e.g., 20 subjects
[4,5]; 31 subjects [6]; 29 subjects [3]; and 50 subjects [8]. In order to assess large scale performance, the methods need to be tested on comparatively larger data size.

\subsection{Heritability of ECG Waves}

The heritability analysis of ECG has shown intraclass correlation between adult male twins (monozygotic and dizygotic). In particular, ventricular repolarization and heart rate are significant heritable components while composite $Q, R, S$ waves do not show a significant heritable component [18].

\subsection{Customization of ECG-Enabled Biometric System}

A customized ECG-enabled biometric system similar to an ECG analyzer which diagnoses cardiac ailments online is needed that can perform the authentication task across a range of conditions. The size of such a device is as small as a mobile phone so that it could be kept in the pocket and performs the authentication task during an individual's normal activity.

\section{Conclusions}

This paper has evaluated the feasibility of ECG as a biometric for individual authentication and proposed a method for ECG-enabled biometric authentication system. Unlike conventional biometrics that are neither secrets nor robust enough against falsification, ECG is inherited to an individual which is highly secure and impossible to be forged. Most importantly, ECG has an inherent real-time feature of vitality signs which ensures that an ECG cannot be acquired unless the person is not live or it can not be acquired unless the person to be authenticated is not present at the authentication desk. Therefore, it is robust enough against the falsified credentials to be enrolled in the system. We have shown that ECG has potential to provide an excellent source of supplementary information in a multibiometric system. The fusion of ECG with the face biometric and with the fingerprint biometric has shown a significant improvement in authentication performance of both of the fused systems. In addition, we have critically examined the research concerns of ECG-enabled biometric authentication system across wide range of conditions.

The laboratory demonstration of the biometric use of ECG has shown great promise, but the fruitful directions for further research include the following: 1) The ECGenabled biometric system must perform the authentication task across wide range of conditions over a larger population including the data acquired at larger time intervals. 2) It is important to discover that up to what extent an ECG varies under different anxiety levels. An investigation of robustness to the subjects of different 
emotional states is still needed to validate the results of most methods. 3) The analysis methods of ECG signal is still in its infancy. The exploration of alternative classification techniques that are robust to handle variations in the features present that is to be claimed. 4) Specific efforts are needed to check the quality of the signals which is acquired abruptly. It may be because of noncooperation of the user or the presence of some artifacts in the signal. It would support the data representation methods which are working in non-standardized features framework.

\section{REFERENCES}

[1] B. P. Simon and C. Eswaran, "An ECG Classifier Designed Using Modified Decision Based Neural Networks," Computer and Biomedical Research, Vol. 30, No. 4, 1997, pp. 257-272. doi:10.1006/cbmr.1997.1446

[2] S. A. Israel, J. M. Irvine, A. Cheng, M. D. Wiederhold and B. K. Wiederhold, "ECG to Identify Individuals," Pattern Recognition, Vol. 38, No. 1, 2005, pp. 133-142. doi:10.1016/j.patcog.2004.05.014

[3] J. M. Irvin and S. A. Israel, "A Sequential Procedure for Individual Identity Verification Using ECG," EURASIP Journal on Advances in Signal Processing, Vol. 2009, 2009, Article ID: 243215, pp. 1-13.

[4] L. Biel, O. Pettersson, L. Philipson and P. Wide, "ECG Analysis: A New Approach in Human Identification," IEEE Transaction on Instrumentation and Measurement, Vol. 50, No. 3, 2001, pp. 808-812. doi: $10.1109 / 19.930458$

[5] T. W. Shen, W. J. Tompkins and Y. H. Hu, "One-Lead ECG for Identity Verification," Proceedings of the Second Joint EMBS/BMES Conference, Houston, 23-26 October 2002, pp. 62-63.

[6] Y. Wang, F. Agrafioti, D. Hatzinakos and K. N. Plataniotis, "Analysis of Human Electrocardiogram for Biometric Recognition," EURASIP Journal on Advances in Signal Processing, Vol. 2008, 2008, Article ID: 148658, pp. $1-11$.

[7] Y. N. Singh and P. Gupta, "Biometric Method for Human Identification Using Electrocardiogram," Proceedings of the 3rd IAPR/IEEE International Conference on Biometrics, ICB 2009, LNCS, Springer-Verlag, Berlin, Vol.
5558, 2009, pp. 1270-1279.

[8] Y. N. Singh and P. Gupta, "Correlation Based Classification of Heartbeats for Individual Identification," Journal of Soft Computing, Vol. 15, No. 3, 2011, pp. 449-460. doi:10.1007/s00500-009-0525-y

[9] F. Sufi and I. Khalil, "An Automated Patient Authentication System for Remote Telecardiology," Proceedings of the Fourth International Conference on Intelligent Sensors, Sensor Networks and Information Processing, ISSNIP 2008, 15-18 December 2008, pp. 279-284.

[10] J. Pan and W. J. Tompkins, “A Real Time QRS Detection Algorithm," IEEE Transactions on Biomedical Engineering, Vol. 33, No. 3, 1985, pp. 230-236. doi:10.1109/TBME.1985.325532

[11] Y. N. Singh and P. Gupta, "A Robust Delineation Approach of Electrocardiographic P Waves," Proceedings of the 2009 IEEE Symposium on Industrial Electronics and Applications, ISIEA 2009, Vol. 2, 2009, pp. 846-849.

[12] Y. N. Singh and P. Gupta, "A Robust and Efficient Technique of T Wave Delineation from Electrocardiogram," Proceedings of the 2nd International Conference on Bio-Inspired Systems and Signal Processing, BIOSIGNALS, 2009, pp. 146-154.

[13] H. C. Bazett, "An Analysis of the Time-Relations of Electrocardiograms," Heart, Vol. 7, 1920, pp. 353-370.

[14] R. O. Duda, P. E. Hart and D. G. Stork, "Pattern Classification," 2nd Edition, Wiley, New Delhi.

[15] P. Verlinde, P. Druyts, G. Cholet and M. Acheroy, “Applying Bayes Based Classifiers for Decision Fusion in a Multi-Modal Identity Verification System," Proceedings of the International Symposium on Pattern Recognition in Memoriam Pierre Devijver, Brussels, 12 February 1999.

[16] Physionet, "Physiobank Archives," Massachusetts Institute of Technology, Cambridge, 2011. http://www.physionet.org/physiobank/database/\#ecg

[17] "Biometric Score Set," National Institute of Standard and Technology, 2011. http://www.itl.nist.gov/iad/894.03/biometricscores/

[18] M. W. Russell, I. Law, P. Sholinsky and R. R. Fabsitz, "Heritability of ECG Measurements in Adult Male Twins," Journal of Electrocardiology, Vol. 30, No. 1, 1998, pp. 64-68. doi:10.1016/S0022-0736(98)80034-4 